\title{
Regulation of erythroid differentiation by miR-376a and its targets
}

\author{
Fang Wang ${ }^{1}$, Jia Yu ${ }^{1}$, Gui-Hua Yang ${ }^{1}$, Xiao-Shuang Wang $^{1}$, Jun-Wu Zhang ${ }^{1}$ \\ ${ }^{I}$ Department of Biochemistry and Molecular Biology, National Laboratory of Medical Molecular Biology, Institute of Basic Medi- \\ cal Sciences, Chinese Academy of Medical Sciences and Peking Union Medical College, 5 Dong Dan San Tiao, Beijing 100005, \\ China
}

Lineage differentiation is a continuous process during which fated progenitor cells execute specific programs to produce mature counterparts. This lineage-restricted pathway can be controlled by particular regulators, which are usually exclusively expressed in certain cell types or at specific differentiation stages. Here we report that miR376a participates in the regulation of the early stages of human erythropoiesis by targeting cyclin-dependent kinase 2 (CDK2) and Argonaute 2 (Ago2). Among various human leukemia cell lines, miR-376a was only detected in K562 cells which originated from a progenitor common to the erythroid and megakaryotic lineages. Enforced expression of miR-376a or silencing of $C D K 2$ and $A g o 2$ by RNAi inhibits erythroid differentiation of K562 cells. Hematopoietic progenitor cells transduced with miR-376a showed a significant reduction of their erythroid clonogenic capacity. MiR-376a is relatively abundant in erythroid progenitor cells, where it reduces expression of CDK2 and maintains a low level of differentiation due to cell cycle arrest and decreased cell growth. Following erythroid induction, miR376a is significantly down-regulated and CDK2 is released from miR-376a inhibition, thereby facilitating the escape of progenitor cells from the quiescent state into erythroid differentiation. Moreover, our results establish a functional link between miR-376a and Ago2, a key factor in miRNA biogenesis and silencing pathways with novel roles in human hematopoiesis.

Keywords: microRNA-376a; cyclin-dependent kinase 2 (CDK2); Argonaute 2 (Ago2); erythroid differentiation Cell Research (2011) 21:1196-1209. doi:10.1038/cr.2011.79; published online 10 May 2011

\section{Introduction}

Normal erythropoiesis in adult humans generates about $10^{11}$ new red blood cells each day through the commitment of hematopoietic stem cells into erythroid progenitors, which subsequently differentiate into mature red cells [1-3]. The resulting number of fully differentiated red cells is precisely controlled to match physiologic and developmental needs through a multi-stage process regulated by complex intercellular networks. Understanding the regulatory mechanisms of erythroid differentiation is of crucial importance to developing methods for the manipulation of HSCs for use in the treatment of

Correspondence: Jun-Wu Zhang

Tel: 86-10-65296423; Fax: 86-10-65240529

E-mail: junwu_zhang@pumc.edu.cn

Received 26 July 2010; revised 23 November 2010; accepted 26 January 2011; published online 10 May 2011 anemia.

MicroRNAs (miRNAs) are a class of endogenous molecules that post-transcriptionally regulate target gene expression [4-6]. In many cases, the identification of specific targets of miRNAs has highlighted important cellular events and demonstrated that aberrant microRNA expression is a common feature of many diseases [7-9]. Previously, several miRNAs have been identified as being involved in hematopoiesis through their target genes. For example, miR-223 enhances retinoic acid (RA)induced granulocytic differentiation by targeting nuclear factor I/A (NFIA) [10]; miR-155 transduction greatly reduces both the myeloid and erythroid colony formation of normal human hematopoietic stem-progenitor cells [11]; miR-221 and miR-222 inhibit normal erythropoiesis and erythroleukemic cell growth via kit protein down-regulation [12]; miR-451 and GATA transcription factors were shown to comprise a regulatory circuit that modulates erythroid maturation [13-15]; miR-210 is 
involved in increased expression of the $\gamma$-globin gene in differentiating erythroid cells [16]; and the up-regulation of the let-7 miRNA family in circulating erythroid cells is associated with the fetal-to-adult hemoglobin switching during human development [17]. Our previous work has identified two regulatory miRNAs, miR-223 and miR-103, that inhibit erythroid differentiation through targeting LIM domain only 2 (LMO2) $[18,19]$ and forkhead box J2 (FoxJ2) [20], respectively. Even considering these findings, relatively little is known about the roles of this class of regulatory molecule in blood cell differentiation, and additional of miRNAs involved in hematopoiesis still need to be identified. Moreover, one miRNA could target multiple proteins, thereby making the analysis of a single miRNA an efficient method for clarifying the complex connections involved in the regulatory networks of hematopoietic differentiation.

Clustered miRNAs had been profiled in leukemia cell lines that represent different hematopoietic lineages [21]. Among them, miR-376a drew our attention because it was restrictedly expressed in the K562 line, which originated from a progenitor common to the erythroid and megakaryotic lineages, but not in the other leukemic cell lines. Since K562 can undergo erythroid differentiation upon hemin induction, we performed a functional analysis of miR-376a in K562 cells. Enforced expression of miR-376a blocked erythroid differentiation by targeting

Ago2 and CDK2. More importantly, the lentiviral vectormediated transduction of miR-376a into CD34+ stem/ progenitor cells (HPCs) delayed erythrocyte maturation in erythroid cultures and prevented erythroid colony formation in a semisolid medium. Our study demonstrates that miR-376a functions as a negative regulator in human erythropoiesis, a finding that should enable the identification of additional novel mechanisms of normal hematopoiesis, as well as leukemogenesis.

\section{Results}

$A$ rapid decrease in miR-376a expression occurs in the early stages of erythropoiesis

Previously, our study examining the organization and expression of miRNA clusters showed that miR-376a was specifically expressed in the K562 line, a myelogenous leukemia cell line with potential to megakaryocyte and erythroid differentiation [21]. Following this work, we performed another northern blot to characterize the expression of miR-376a in twelve different leukemia cell lines representing five hematopoietic lineages and found that miR-376a expression was indeed specific to K562 cells, and it was not expressed in the other eleven hematopioetic cell lines examined, including HEL, which is usually thought to be unipotent erythroid progenitors (Figure 1A). Additionally, a human multiple-tissue ex-

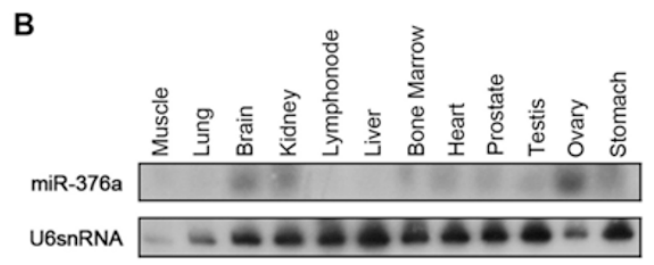

C

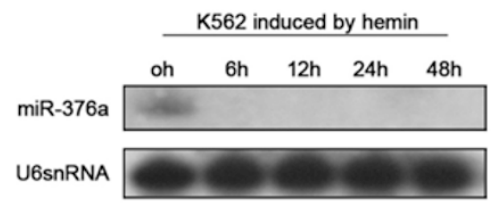

D

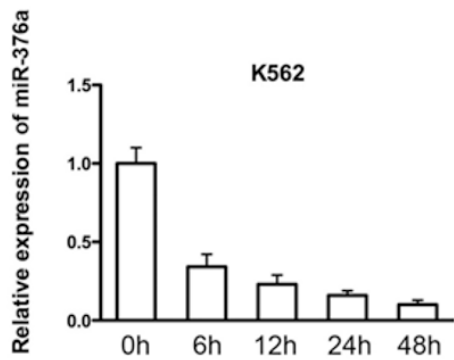

E

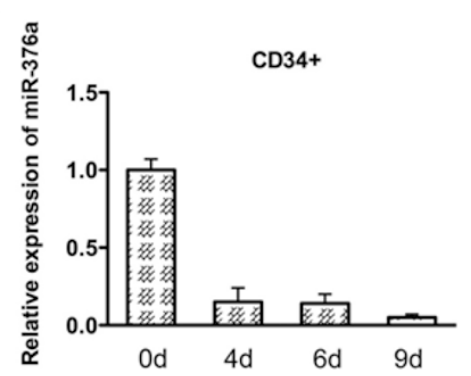

Figure 1 Detection of miR-376a expression in various cell lines and tissues and during erythroid differentiation. (A) Northern blot analysis of various leukemia cell lines showing miR-376a expression is K562-specific. U6 snRNA is a loading control. (B) Northern blot analysis of miR-376a expression in multiple human tissues. (C-D) Northern blot analysis and quantitative realtime PCR showing a marked decrease of miR-376a levels in K562 cells after treatment with hemin for 6, 12, 24, and 48 h. (E) Quantitative real-time PCR analysis of miR-376a expression in CD34+ HPCs induced into erythroid differentiation for 4, 6 , and 9 days. Comparative miRNA real-time PCR was performed in triplicate and expression levels were normalized to U6 snRNA. Error bars represent standard deviation. 
pression profile showed that miR-376a was relatively widely expressed in several tissues (Figure 1B). Since miR-376a is detectable in K562 cells and K562 is more potent than HEL, we hypothesized that miR-376a may function in early erythroid differentiation. To test this prediction, we induced K562 cells into erythroid differentiation with hemin and measured miR-376a expression level by northern blotting. A significant reduction in miR-376a was detected in K562 cells after 6 hours of hemin treatment (Figure 1C). Similar results were obtaind by quantitative real-time PCR (q-RT PCR) (Figure 1D). Furthermore, we isolated human CD34+ HPCs from human cord blood and induced them into erythroid differentiation. Following 4 days of induction, miR-376a expression was markedly down-regulated (Figure 1E). Taken together, these findings suggested that miR-376a might act as a negative modulator of early erythropoiesis.
Enforced expression of miR-376a inhibits erythroid differentiation

To examine whether miR-376a affects erythroid differentiation, K562 cells were transfected with either premiR-376a or a negative control. Twenty-four hours following transfection, the cells were treated with hemin to induce erythroid differentiation, and then collected at 12 , 24,48 , and $72 \mathrm{~h}$ post-induction. In K562 cells transfected with the negative control, miR-376a immediately disappeared after the addition of hemin (Supplementary information, Figure S1A). However, a significant increase in the level of miR-376a was detected at each time point in cells treated with pre-miR-376a, which demonstrated successful transfection and expression of pre-miR-376a (Supplementary information, Figure S1A).

Benzidine staining was performed to identify hemoglobin-containing cells, which are representative of differentiated erythrocytes. Our results showed that over-
A

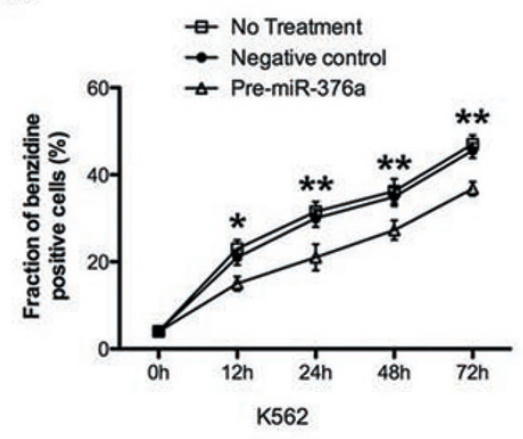

B

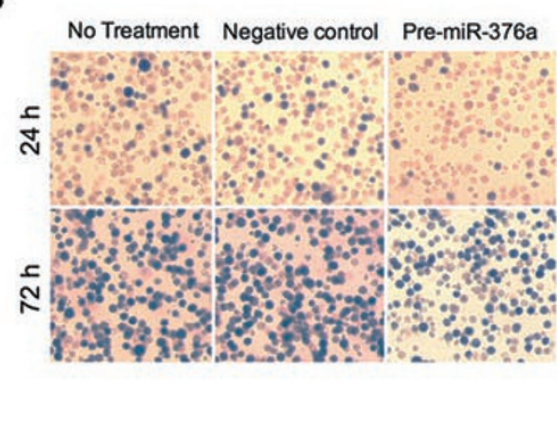

C

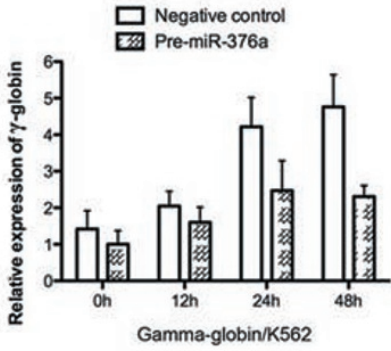

G

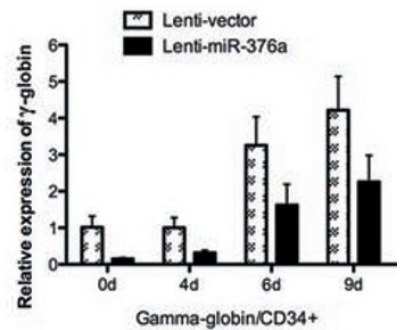

Figure 2 Over-expression of miR-376a inhibits the erythroid differentiation of K562 cells and CD34+ HPCs. (A) Benzidine staining of $\mathrm{K} 562$ cells that were untransfected, or transfected with a negative control precursor (negative control) or a miR376 a precursor (Pre-miR-376a) were induced with hemin for 12, 24, 48, and $72 \mathrm{~h}$. Pre-miR-376a reduces the percentage of benzidine-positive cells at different time points. The fraction of benzidine-positive cells was determined by a benzidine cytochemical test. Error bars represent the standard deviation and were obtained from three independent experiments. * indicates $P<0.05,{ }^{* *}$ indicates $P<0.01$. (B) Representative benzidine staining of un-transfected and transfected K562 cells following 24 and $72 \mathrm{~h}$ of hemin induction. (C-D) Quantitative real-time PCR analysis of $\gamma$-globin and CD235A in the transfected K562 cells after hemin induction for 12,24 , and $48 \mathrm{~h}$. Real-time PCR analysis was performed in triplicate and the expression levels of $\gamma$-globin and CD235A mRNAs were normalized to GAPDH mRNA. Error bars represent standard deviation. (E) Quantitative real-time PCR analysis of miR-376a in the CD34+ HPCs that were infected with a lenti-virus expressing mature miR-376a (lenti-miR-376a) or a control virus (lenti-vector) and cultured in erythroid induction medium for the indicated time. (F-G) Quantitative real-time PCR analysis of $\gamma$-globin and CD235A in the CD34+ HPCs infected with the recombinant viruses and cultured in erythroid induction medium for the indicated time. 
expression of pre-miR-376a reduced the proportion of benzidine-positive cells following hemin-induced K562 erythroid differentiation (Figure 2A and 2B). Additionally, a significant inhibition of $\gamma$-globin expression (Figure 2C), accompanied by reduced expression of CD235a (Figure 2D), was observed in K562 cells transfected with pre-miR-376a as compared to cells transfected with the negative control. These results suggested that the enforced expression of miR-376a suppressed the hemininduced erythroid differentiation of K562 cells. We also conducted a miRNA loss-of-function study using a miR376a inhibitor (Anti-miR-376a). The level of endogenous miR-376a was reduced after treatment with Anti-miR376a (Supplementary information, Figure S1B). K562 cells treated with Anti-miR-376a showed an increase in hemoglobin accumulation, as indicated by an increase in benzidine-positive cells (Supplementary information, Figure S2). These results suggest that the inhibition of miR-376a expression promotes the hemin-induced erythroid differentiation of K562 cells.

To further assess the role of miR-376a during normal erythroid differentiation, we transduced CD34+ HPCs with recombinant lentivirus harboring miR-376a (lentimiR-376a) and recombinant lentivirus harboring an empty vector (lenti-vector) respectively. Twenty-four hours following transduction, the cells were grown in an erythroid culture medium to drive their selective differentiation. After 4 days in the erythroid induction culture medium, the expression level of miR-376a in the lentimiR-376a-infected cells was about 4-fold higher than in the lenti-vector-infected cells (Figure 2E). Similar results were observed at 6 and 9 days. As shown in Figure 2F and $2 \mathrm{G}$, at each time point examined, a marked decrease in CD235a and $\gamma$-globin expression was detected in CD34+ cells treated with lenti-miR-376a as compared to cells treated with lenti-vector. These results suggest that the enforced expression of miR-376a in CD34+ cells suppresses the erythroid differentiation of CD34+ HPCs.

\section{miR-376a targets Ago2 and CDK2}

It is well known that miRNAs function by binding to specific mRNA targets and interfering with their stability or repressing their translation. As a beginning step towards identifying the putative targets of miR-376a, we performed a bioinformatic screen for mRNAs whose $3^{\prime}$ UTRs contained sequences complementary to miR-376a. Results obtained from three different algorithms (TargetScan, miRanda and PicTar) varied. We considered all candidates related to erythropoiesis that were predicted by these programs. Ago2 was one of the few predictions identified by all three programs and that scored relatively high. Recently, experimental evidence indicated that
Ago2 controls the early development of mouse erythroid cells in vivo [22-23]. Hematopoietic reconstitution with Ago $2^{-/}$bone marrow cells showed a dramatic accumulation of immature erythroid precursors and a decrease in mature erythroblasts [22]. CDK2, a cyclin-dependent kinase promoting G1/S transition, was another miR-376a target candidate. Previously, investigators have suggested that CDK2 contributes to the expansion phase at an early stage of erythroid differentiation from CD34+ cells, and its temporal up-regulation is necessary for the beginning period of erythropoiesis [24]. The rapid proliferation of erythroblasts may be due to the rapid transition of cells from $\mathrm{G} 1$ to $\mathrm{S}$ phase [24].

Bioinformatic analysis predicted that the seed sequence of miR-376a matches Ago 2 mRNA nucleotides 2911 to 2917 and CDK2 mRNA nucleotides 2192 to 2 198, which are conserved in multiple mammals (Figure $3 \mathrm{~A}, 3 \mathrm{~B})$. To validate the results of our screening, we first determined the effect of miR-376a on reporter constructs carrying the entire 3' UTR of Ago2 (Ago2_WT) and $C D K 2$ (CDK2_WT) mRNAs (Figure 3A and 3B) by luciferase reporter assay in Hela cells. As expected, the luciferase activities of Ago2 WT and CDK2 WT were remarkably down-regulated in Hela cells transfected with pre-miR-376a, while the luciferase activities were unaffected in cells transfected with the negative control (Figure 3C, 3D and Supplementary information, Figure S2). In contrast, the luciferase activities of Ago2_WT and CDK2 WT were up-regulated in K562 cells treated with Anti-miR-376a (Supplementary information, Figure S3). Furthermore, we generated two other luciferase constructs bearing mutated nucleotides at the putative target sites in the 3' UTRs of Ago2 (Ago2_MUT) and CDK2 (CDK2_MUT) (Figure 3A and 3B). The mutations prevented miR-376a from interfering with luciferase activity, indicating that the 3' UTRs of Ago2 and CDK2 are indeed targets of miR-376a (Figure 3C and 3D).

We then explored whether the endogenous Ago2 and CDK2 in K562 cells were affected by miR-376a. To this aim, qRT-PCR and western blot analysis were performed to measure the mRNA and protein expression levels of the two gene products after cell transfection with the microRNA. Transfection of K562 cells with pre-miR$376 \mathrm{a}$ led to a marked reduction in the protein expression levels of Ago2 and CDK2 (Figure 3E), whereas almost no effect was observed on the mRNA levels (Figure 3F and Supplementary information, Figure S4). Conversely, the protein expression levels of Ago2 and CDK2 were significantly increased after endogenous miR-376a was blocked with miR-376a inhibitor (Figure $3 \mathrm{G}, 3 \mathrm{H}$ and Supplementary information, Figure S4). Considered together, these results provide experimental support for 
A

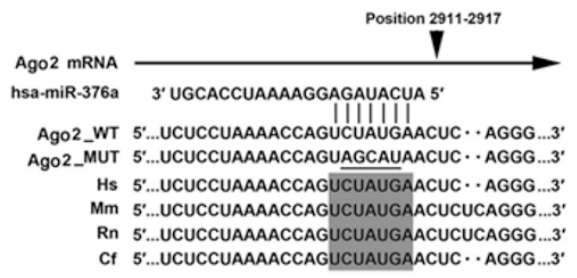

B

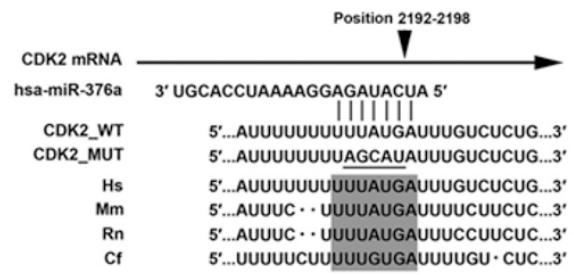

C

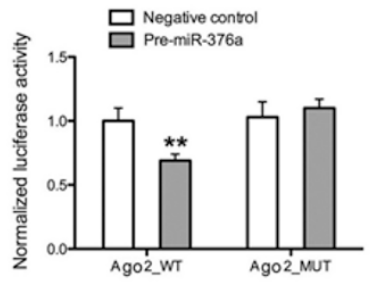

D

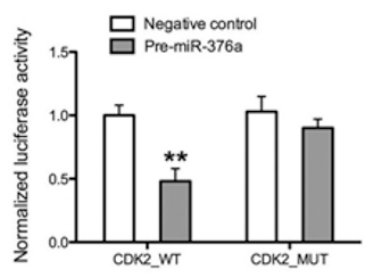

E

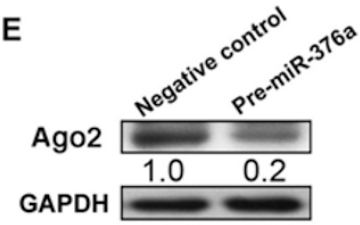

F

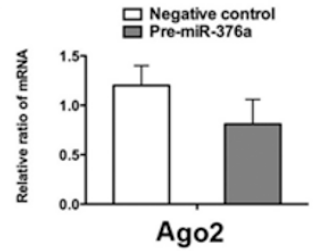

G

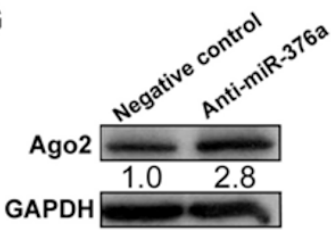

H

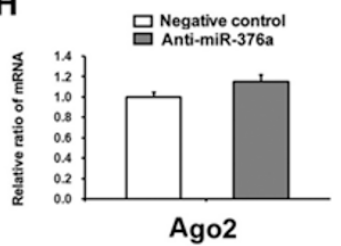

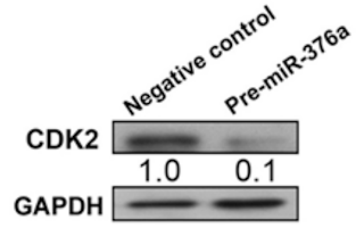

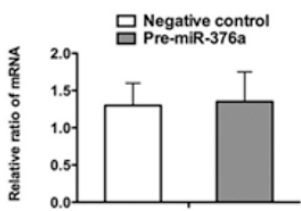

CDK2
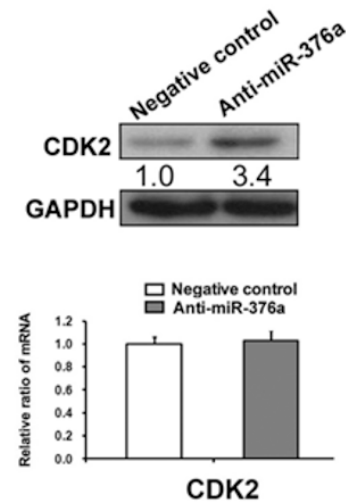

Figure 3 Ago2 and CDK2 are targets of miR-376a. (A-B) Sequences of the miR-376a binding sites within the human Ago2 and CDK2 3' UTRs and schematic reporter constructs. Shaded areas represent the conserved complementary nucleotides of the miR-376a seed sequence in various mammals (Hs, human; Mm, mouse; Rn, rat; Cf, chicken). Ago2_WT and CDK2_WT represent the reporter constructs containing the entire 3' UTR sequences of Ago2 and CDK2. Ago2_MUT and CDK2_MUT represent the reporter constructs containing mutated nucleotides. (C-D) Luciferase activities of Ago2_WT and CDK2 WT showing that pre-miR-376a inhibits the luciferase activity of these two constructs as compared with their respective mutated constructs. Error bars are derived from three experiments in triplicate. ${ }^{* *}$ indicates $P<0.01$. (E) Immunoblotting for Ago2 and CDK2 in extracts from K562 cells transfected with pre-miR-376a showed an obvious decrease in protein expression $48 \mathrm{~h}$ after cell transfection. (F) Relative expression of Ago2 and CDK2 in total RNA from K562 cells transfected with pre-miR-376a (gray bars) or negative control (blank bars) was analyzed $48 \mathrm{~h}$ after cell transfection. (G) Immunoblotting for Ago2 and CDK2 in extracts from K562 cells transfected with Anti-miR-376a showed an obvious increase in protein expression $48 \mathrm{~h}$ after cell transfection. The signal in each lane was quantified using ImageJ software and the ratio of Ago2 or CDK2 to GAPDH was determined. $(\mathrm{H})$ The relative expression of Ago2 and CDK2 in total RNA from K562 cells transfected with Anti-miR-376a or negative control was analyzed $48 \mathrm{~h}$ after cell transfection. GAPDH was used as an internal control. The specificity of each reaction in real-time PCR was assessed with a melting curve and $t$-test. All data are shown as mean \pm S.D. and are based on three independent experiments.

the idea that Ago2 and CDK2 expression are negatively regulated by miR-376a in erythroid cells.

Over-expression of miR-376a results in the arrest of erythroid differentiation due to Ago2 down-regulation

The requirement for Ago2 in mouse erythroid development suggests the possibility that Ago 2 may also function in human erythroid differentiation [22]. To examine the effect of Ago2 in human erythropoiesis, we first con- firmed by western blot analysis that the expression level of Ago2 was significantly increased in K562 cells after hemin induction (Figure 4A), whereas the Ago2 mRNA level was not affected by hemin treatment (Supplementary information, Figure S5). Thus, we reasoned that interfering with Ago2 expression in human erythroid progenitor cells (such as K562) might have inhibitory effect on differentiation. As expected, the reduction of Ago2 level (Figure 4B) caused by small interfering RNA (si_Ago2) 
A

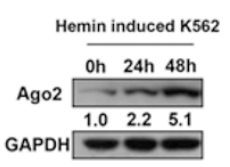

B

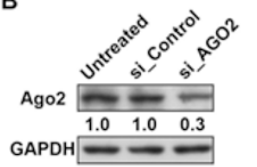

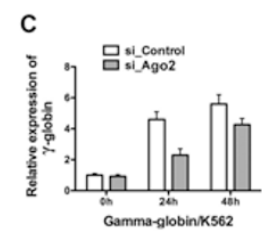

D

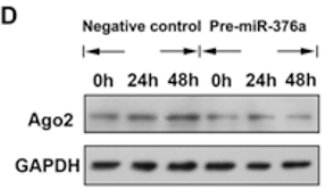

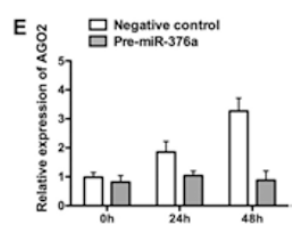

$\mathrm{F}$

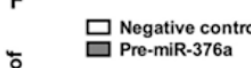

$\div \quad$ Pre-mir-376a

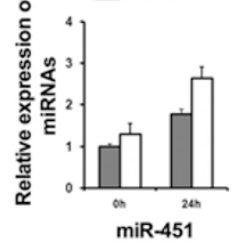

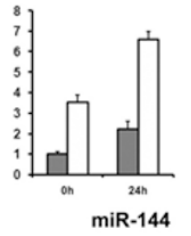

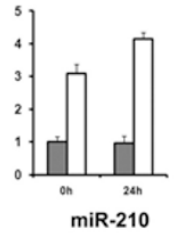

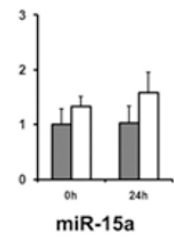

G

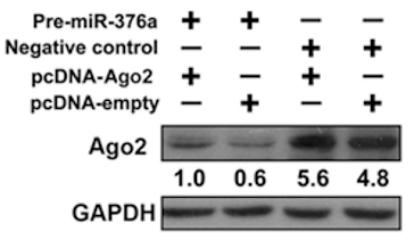

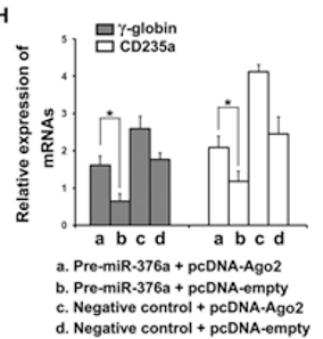

Figure 4 MiR-376a represses the erythroid differentiation of K562 cells through directly targeting Ago2. (A) Immunoblotting of Ago2 in extracts from K562 cells that were induced by hemin for 24 and $48 \mathrm{~h}$. GAPDH serves as a loading control. The signal in each lane was quantified using ImageJ software and the ratio of Ago2 to GAPDH was determined. (B) Immunoblots of Ago2 and GAPDH in extracts from K562 cells $48 \mathrm{~h}$ after transfection with a control siRNA (si_Control) or a siRNA for Ago2 (si_ Ago2). The results showed a significant decrease in Ago2 expression in the cells transfected with si_Ago2. (C) Quantitative real-time PCR analysis of $\gamma$-globin expression in K562 cells induced by hemin for 24 and $48 \mathrm{~h}$ after cell transfection with si Control or si_Ago2. Comparative real-time PCR was performed in triplicate and the expression level of $\gamma$-globin mRNA was normalized to GAPDH mRNA. Error bars represent standard deviation. (D) Immunoblotting of Ago2 and GAPDH in extracts from K562 cells induced by hemin for 24 and $48 \mathrm{~h}$ after treatment with pre-miR-376a or the negative control. (E) Normalized expression of Ago2 in D by ImageJ software. (F) Quantitative real-time PCR analysis of miR-451, 144, 126, 210, and 15a expression in K562 cells following pre-miR-376a or negative control transfection for $24 \mathrm{~h}$. (G) Immunoblotting of Ago2 and GAPDH in extracts from K562 cells that were transfected with pre-miR-376a or the negative control for $24 \mathrm{~h}$ and then subsequently treated for another $48 \mathrm{~h}$ with different combinations of pcDNA-Ago2 or pcDNA-empty, and pre-miR-376a or negative control precursor. (H) Quantitative real-time PCR analysis of $\gamma$-globin and CD235A expression in K562 cells that were transfected with pre-miR-376a or the negative control for $24 \mathrm{~h}$, subsequently treated for another $24 \mathrm{~h}$ with either pcDNA-Ago 2 or pcDNA-empty, and then exposed to hemin induction for $48 \mathrm{~h}$. * indicates $P<0.05$.

transfection resulted in a marked decrease in $\gamma$-globin expression (Figure 4C), indicating that down-regulation of Ago2 inhibits the proper differentiation of human erythrocytes.

Having established the role of Ago2 in erythroid differentiation, we next investigated the mechanism by which miR-376a regulates erythroid differentiation via Ago2. Ago2 demonstrates nucleolytic activity in siRNA and miRNA biogenesis $[25,26]$. To date, two labs have reported a dicer-independent miRNA biogenesis pathway that requires Ago2 catalysis [27, 28]. Therefore, the observation that Ago 2 modulates erythroid differentiation can be partly explained by its involvement in miRNA activity, especially of those miRNAs involved in erythropoiesis. Therefore, we explored whether an increase of miR-376a was able to disturb the expression of other endogenous miRNAs, such as miR-451, miR-144, miR210, and miR-15a, through the down-regulation of Ago2. The first three of these selected miRNAs were previously experimentally validated as promoters of erythroid differentiation [13-16]. Indeed, the levels of miR-451,
miR-144, and miR-210 were markedly reduced in premiR-376a-transfected K562 cells during hemin-induced erythroid differentiation (Figure 4D-4F). To clarify whether the down-regulation of these miRNAs was associated with the decrease in Ago2 levels caused by miR376a, we measured the expression of miRNAs in K562 cells treated with si_Ago2. Similarly, silencing of Ago2 led to decreased miR-451, miR-144, and miR-210 levels (Supplementary information, Figure S6). Taken together, we believe these results indicate that one of the mechanisms by which miR-376a inhibits erythroid differentiation is by modulating Ago2 levels and blocking the upregulation of certain miRNAs.

Although the functional significance of miR-376a and Ago2 with respect to erythroid differentiation has been clarified, and the regulatory interaction between them has been proposed, the direct relationships between miR-376a, its targets (such as Ago2), and cell phenotype remain unclear. Previous studies adopted a 'rescue' methodology to validate whether a miRNA is able to affect downstream signal pathways by the direct regula- 
tion of its targets [29]. We chose this strategy in order to examine the functional relevance of the miR-376a/ AGO2 interaction in erythroid differentiation. K562 cells were transfected with two constructs, one containing the entire Ago 2 cDNA sequence (pcDNA-Ago2) and one, the miR-376a precursor (pre-miR-376a). Additional cells were transfected with the respective negative controls in place of one or both of these constructs, as follows: (i) pre-miR-376a and pcDNA-Ago2; (ii) pre-miR-376a and pcDNA-empty; (iii) scrambled dsRNAs (microRNA negative control) and pcDNA-Ago2; (iv) scrambled dsRNAs and pcDNA-empty. We also determined that optimal results (i.e. the most significant change in Ago2 levels) are achieved when co-transfection of the two constructs follows a previous treatment with pre-miR$376 \mathrm{a}$ or the negative control. As expected, Ago2 protein levels were observed to be highest in cells co-transfected with scrambled dsRNAs and pcDNA-Ago2 (Figure 4G, lane 3), moderate in cells co-transfected with pre-miR376a and pcDNA-Ago2 (Figure 4G, lane 1), and lowest in cells co-transfected with pre-miR-376a and pcDNAempty (Figure 4G lane 2). In other words, down-regu- lation of Ago 2 by the initial introduction of pre-miR376a was enhanced by pre-miR-376a re-introduction (Figure 4G, lane 2), whereas it was successfully rescued by pcDNA-Ago 2 transfection (Figure 4G, lane 1). Interestingly, the expression levels of two erythroid marker genes, $\gamma$-globin and CD235a, were correlated with the level of Ago2 in K562 cells; that is, introduction of Ago2 to rescue its silencing by pre-miR-376a treatment led to enhanced differentiation (Figure 4H). Furthermore, the erythroid-related miRNA (such as miR-451 and miR144) levels were also increase when Ago 2 was rescued (Supplementary information, Figure S7). Since the catalytic activity of Ago2 is known to be essential to mature miR-451 expression [27], we also constructed a catalytically dead Ago2 (pcDNA-Ago2_Mut) with a substitution of alanine for the aspartate residue in the catalytic DDH motif. We performed a similar "rescue" assay with the inclusion of pcDNA-Ago2_Mut, and miR-451 showed the most significant reduction in transfection groups containing pcDNA-Ago2_Mut when compared to pcDNAAgo2_WT, suggesting the requirement of Ago2 catalysis for miR-451 biogenesis (Supplementary information,
A

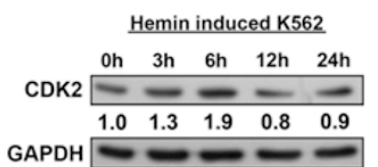

E

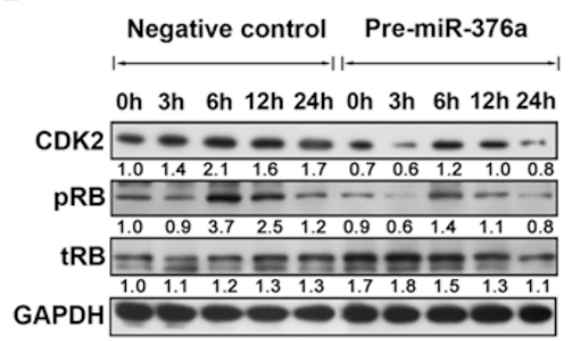

B

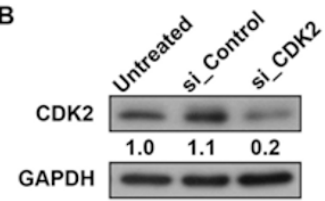

C

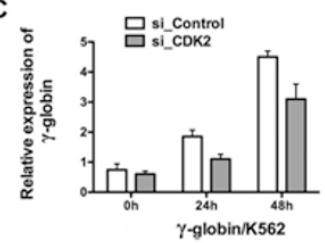

F

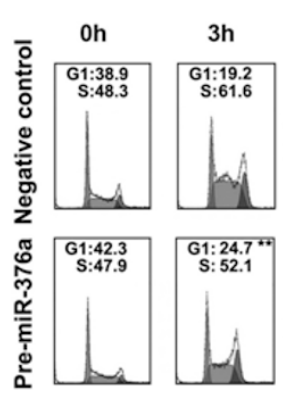

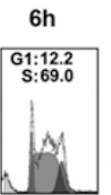
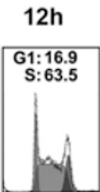

$24 \mathrm{~h}$
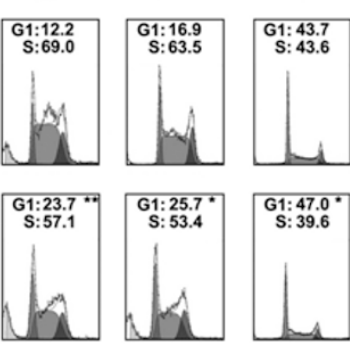

Figure 5 MiR-376a inhibits erythroid proliferation by targeting CDK2 in early erythroid differentiation. (A) Immunoblotting of CDK2 in extracts from K562 cells that were induced with hemin for 3, 6, 12, and $24 \mathrm{~h}$. GAPDH serves as a loading control. (B) Immunoblotting of CDK2 and GAPDH in extracts of K562 cells $48 \mathrm{~h}$ after transfection with a control siRNA (si_Control) or a siRNA for CDK2 (si_CDK2). The results showed a decrease in CDK2 expression in the cells transfected with si_CDK2. (C) Quantitative real-time PCR analysis of Y-globin expression in K562 cells induced by hemin for 24 and $48 \mathrm{~h}$ after si_ Control or si_CDK2 transfection. Comparative real-time PCR was performed in triplicate and the expression level of $\gamma$-globin was normalized to GAPDH mRNA. Error bars represent standard deviation. (D) Cell cycle analysis of K562 cells that are uninduced or induced by hemin for $24 \mathrm{~h}$ after $24 \mathrm{~h}$ cell transfection with si_Control or si_CDK2. Inhibitory S phases are seen in the si_CDK-transfected cell cycle image. (E) Immunoblotting of CDK2, phosphorylated RB, total RB, and GAPDH in extracts from K562 cells induced by hemin for 3, 6, 12, and $24 \mathrm{~h}$ after cell transfection with pre-miR-376a or the negative control. The signal in each lane was quantified using ImageJ software and the ratio of CDK2, pRB, and tRB to GAPDH was determined. (F) Cell cycle analysis of K562 cells that are un-induced or induced by hemin for 3, 6, 12, and $24 \mathrm{~h}$ after cell transfection with pre-miR-376a or the negative control. ${ }^{*} \mathrm{P}<0.05$; ${ }^{*} \mathrm{P}<0.01$. 
Figure S8). These findings demonstrate that miR-376a modulates erythroid differentiation by directly downregulating Ago2 expression.

miR-376a represses erythroid cell cycle progression by targeting CDK2

CDK2 is an important kinase involved in the regulation of cell cycle and cell proliferation. It has been shown to be necessary for primary human erythroid differentiation during the expansion stage of erythroid progenitor cells [24]. In K562 cells, the addition of hemin will also trigger a rapid increase in cell number specifically in the early phases of differentiation [30]. We speculated that CDK2 is, to some extent, required for the expansion phase of K562 erythropoiesis. To address this possibility, western blot analysis was used to assess the expression level of CDK2 throughout K562 differentiation. During the first 6 hours of hemin treatment, the CDK2 level was observed to undergo a rapid, transient increase, followed by a decline after 12 hours (Figure 5A). However, the mRNA level of CDK2 was almost unchanged (Supplementary information, Figure S4). This early increase in CDK2 expression is accompanied by reduced miR-376a expression (Figure 1C), suggesting the functional implication of miR-376a and CDK2 in this process. Moreover, the treatment of K562 cells with a small interfering RNA against CDK2 (si CDK2) led to a continuous decrease in the $\gamma$-globin level after hemin addition (Figure 5B and 5C). Loss of erythroid marker accumulation was paralleled by a reduction in cell numbers at $\mathrm{S}$ phase (Figure $5 \mathrm{D})$, suggesting the essential role of CDK2 in regulating cell proliferation during K562 differentiation. Therefore,

A

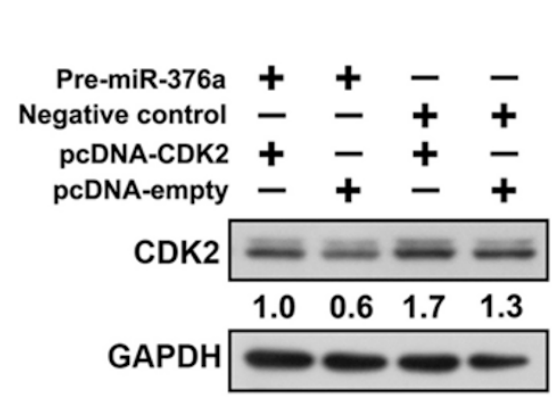

C

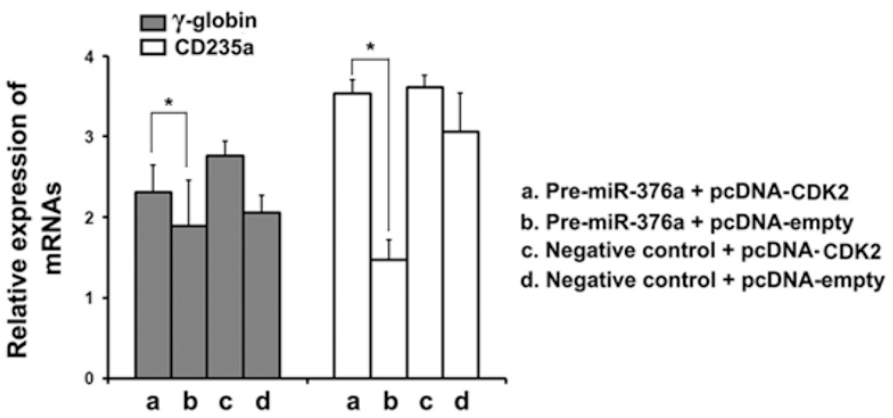

B
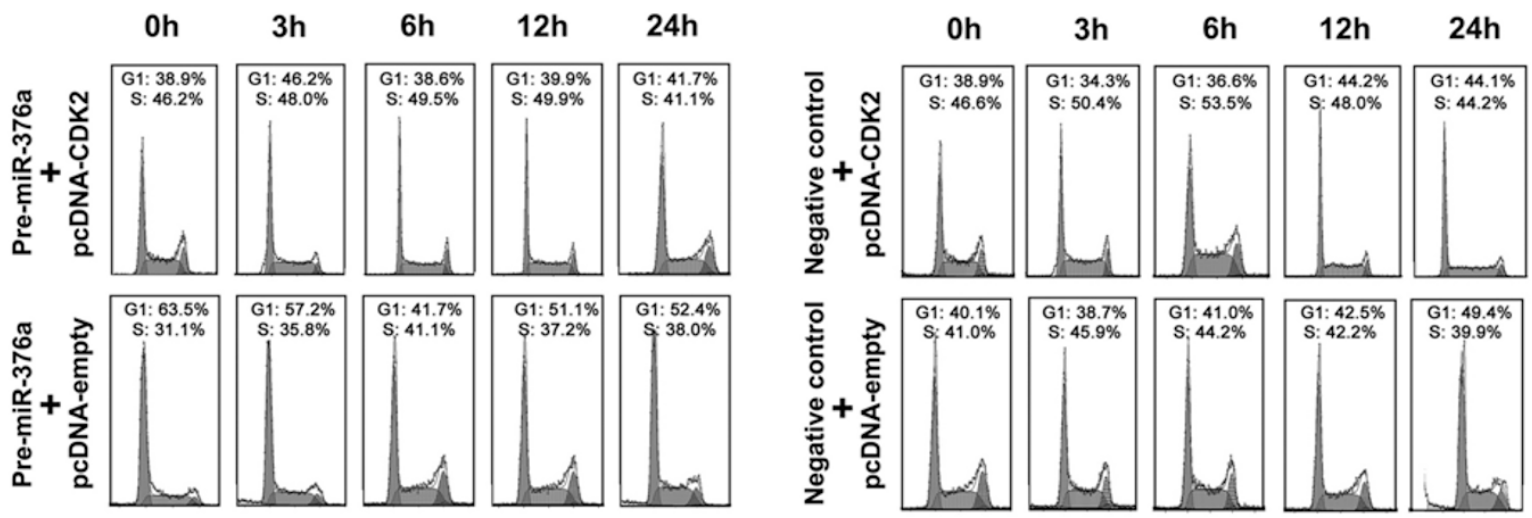

Figure 6 CDK2 is required for miR-376a-directed erythroid differentiation inhibition. (A) Immunoblottings of CDK2 in extracts of K562 cells. The cells were transfected with pre-miR-376a or the negative control. After a $24 \mathrm{~h}$ transfection, the K562 cells were subsequently treated for another $24 \mathrm{~h}$ with different combinations of pcDNA-CDK2 or pcDNA-empty, and pre-miR-376a or the negative control precursor. GAPDH serves as a loading control. (B) Cell cycle analysis of K562 cells. K562 cells were transfected with pre-miR-376a or the negative control for $24 \mathrm{~h}$ and then were treated with different combinations of pcDNACDK2 or pcDNA-empty, and pre-miR-376a or the negative control precursor. This was followed by hemin induction for 3, 6 , 12 , and 24 h. (C) Quantitative real-time PCR analysis of $\gamma$-globin and CD235A in K562 cells that were transfected with premiR-376a or the negative control for $24 \mathrm{~h}$ and then treated with different combinations of pcDNA-CDK2 or pcDNA-empty, and pre-miR-376a or the negative control precursor for anther $24 \mathrm{~h}$. This was followed by hemin induction for $24 \mathrm{~h}$. Comparative real-time PCR analysis was performed in triplicate and the expression levels of $\gamma$-globin and CD235A mRNA were normalized to GAPDH mRNA. Error bars represent standard deviation. * indicates $\mathrm{P}<0.05$. 
A

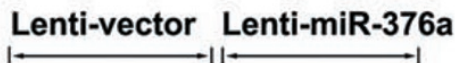

2d 4d 6d 9d 2d 4d 6d 9d

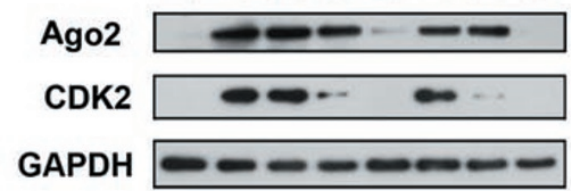

D

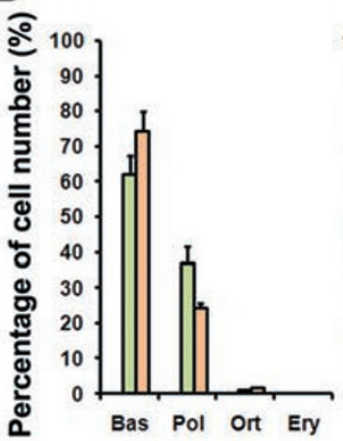

Day 5

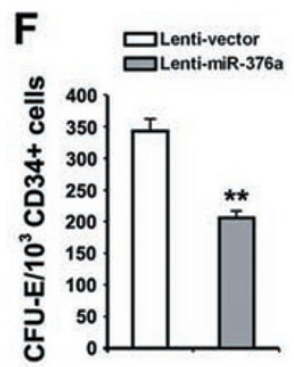

G
B

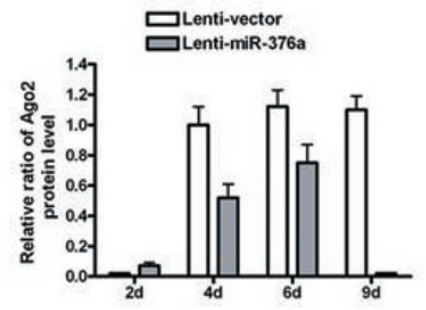

C

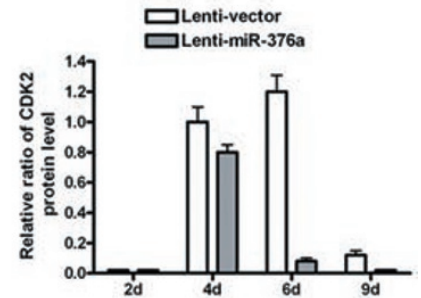

E

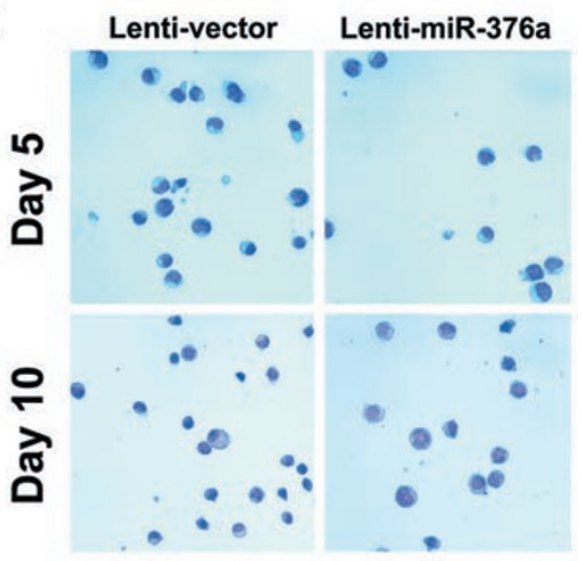

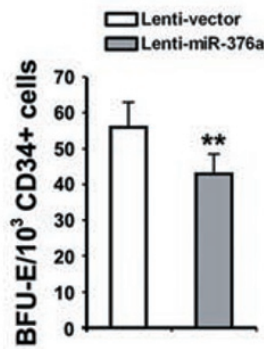

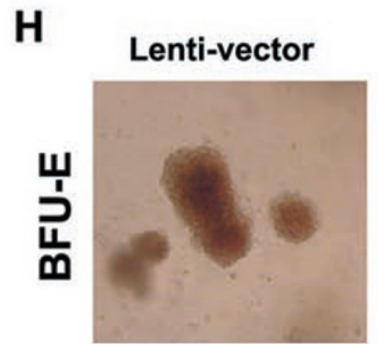

Lenti-miR-376a

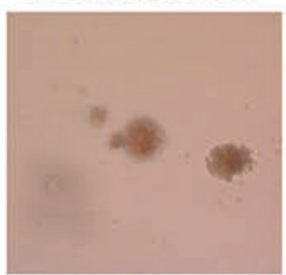

Figure 7 MiR-376a inhibits the erythroid differentiation of CD34+ HPCs. (A) Immunoblotting of Ago2 and CDK2 from extracts taken during the erythroid differentiation of CD34+ HPCs. The CD34+ HPCs were infected with lenti-miR-376a or lenti-vector and exposed to erythroid induction in liquid culture medium. The cells were collected at 2, 4, 6, and 9 days of the culture and used for western blot analysis. GAPDH was also detected for a loading control. (B-C) Normalized expression of Ago2 and CDK2 in $\mathbf{A}$ by ImageJ software. (D) Detection of the degree of erythroid differentiation at days 5,10 , and 15 of the erythroid induction culture of CD34+ HPCs infected with lenti-miR-376a or lenti-vector. Percentages of basophilic (Bas), polychromatophilic (Pol), and orthochromatic (Ort) erythroblasts, and erythrocytes (Ery) were determined by Giemsa staining of cytospin preparations. The results are the mean + S.D. from three independent experiments. (E) Representative Giemsa staining of CD34+ HPCs infected with the viruses at days 5 and 10 of the erythroid induction culture. (F-G) A comparison of the erythroid colony-forming capacity of HPCs transduced with miR-376a and with empty vector. Mean \pm S.D. were obtained from three independent experiments. $(\mathrm{H})$ Representative erythroid-colony forming assays at day 15 of an erythroid induction culture of CD34+ HPCs in semisolid media.

disturbance of the normal cell cycle progression in progenitor cells results in the subsequent inhibition of cell growth and differentiation.

The above observations suggest that a transient increase of CDK2, possibly caused by a reduction in the level of miR-376a, may play a role in driving K562 cells toward erythroid differentiation. To test this possibility, we measured the effects of miR-376a on K562 cell cycle regulation. Consistent with our previous experiments, treatment with pre-miR-376a reduced CDK2 levels and resulted in a concomitant decrease in phosphorylated retinoblastoma $1(\mathrm{RB})$ protein (Figure 5E), and cell cycle arrest at G1/S transition (Figure 5F). Accordingly, premiR-376a treatment inhibited K562 cell growth (Supple- 
mentary information, Figure S9).

Moreover, in a "rescue" assay similar to the one described above, transfection with a pcDNA-CDK2 construct to restore CDK2 levels following pre-miR-376a treatment resulted in increased cell numbers at $\mathrm{S}$ phase (Figure 6A and $6 \mathrm{~B}$ ), accompanied by increased $\gamma$-globin and CD235a expression (Figure 6C). Thus, we conclude that the repression of erythroid differentiation by miR-376a is partially the consequence of impaired cell growth caused by decreased CDK2 expression and delayed G1/S transition.

Over-expression of miR-376a inhibits the erythroid differentiation of HPCs via down-regulation of Ago 2 and CDK2

To assess the physiological relevance of miR-376a and its two targets, Ago2 and CDK2, in normal erythropoiesis, CD34+ HPCs infected with lenti-miR-376a or lentivector (negative control) were allowed to undergo erythroid induction in liquid culture medium and were harvested at the indicated days. As shown in Figure 2E, lenti-miR376a infection resulted in a level of miR-376a expression that was about four times higher than that of HPCs infected with lenti-vector. Accordingly, the protein levels of Ago2 and CDK2 were markedly reduced in lenti-miR-376ainfected cells when compared with the empty vector-treated HPCs (Figure 7A). The quantitative analysis of western blot results is shown in Figure 7B and 7C. In agreement with the results from K562 cells, Ago2 displayed increased expression during CD34+ erythroid differentiation, while CDK2 showed accumulation only in the early stage of differentiation (Figure 7A-7C).

One of the direct roles ascribed to CDK2 is an influence on cell growth. Since miR-376a can reduce CDK2 expression in normal erythropoiesis, we reasoned that overexpression of miR-376a might inhibit cell proliferation in HPCs. As expected, HPCs treated with lenti-miR-376a underwent proliferation arrest as compared with the lentivector-treated cells (Supplementary information, Figure S9). Morphological analysis of primary erythroid cell differentiation showed a significant increase in the proportion of immature erythroblasts (basophilic and polychromatic erythroblasts) in lenti-miR-376a-treated cells and a concomitant decrease in mature erythroblasts (orthochromatic and erythrocyte), as evaluated at different differentiation stages (Figure 7D). Figure 7E shows the representative Giemsa staining analysis of cells after five and ten days of erythroid culture, respectively. The lenti-miR-376a-transduced cells exhibited impaired erythropoiesis as compared with lentivector-treated cells (Figure 7E).

We next performed colony formation assays on methylcellulose medium to evaluate the capacity of miR-376a to regulate primary erythroid differentiation. Lenti-miR- 376a- and lenti-vector-transduced CD34+ cells were plated in semisolid cultures (one day after infection). The numbers of CFU-Es and BFU-Es colonies were scored after 7 and 14 days of culture. The erythrocytic clonogenic capacity of HPCs over-expressing miR-376 was reduced to about $65 \%$ of the control value (Figure $7 \mathrm{~F}$ and $7 \mathrm{G}$ ). Accordingly, HPCs treated with lenti-miR376a formed a significantly less number of colonies than control lenti-vector-treated cells (Supplementary information, Figure S10). Figure $7 \mathrm{H}$ shows the representative micrograph of BFU-Es of HPCs infected with lentimiR-376a after 14 days of culture compared with lentivector-treated cells. The introduction of miR-376a to HPCs caused a dramatic decrease in the size of BFU-E colonies, to an average size of 50 cells per colony (typical BFU-E colony contains 64 or more hemoglobinized cells). Taken together, these results demonstrated an inhibitory effect of miR-376a on normal erythroid differentiation.

\section{Discussion}

Lineage commitment and differentiation are a continuous process during which fated progenitor cells execute specific differentiation programs to produce mature counterparts. Each lineage-restricted pathway can be achieved through the action of several particular regulators, which are exclusively expressed at proper stages of differentiation. Our results show that miR-376a, whose expression is limited to the early stages of erythroid differentiation, down-regulates $A g O 2$ and $C D K 2$, thereby inhibitting progenitor cell growth and differentiation. Several studies have reported a handful of miRNAs that are differentially expressed in CD34+ HPCs or K562 cells following the induction of erythroid differentiation, and that regulate erythroid differentiation through their target mRNAs, such as miR-221, miR-222 [12], miR-451, miR-144 [1315], miR-210 [16], and let-7d [17], etc. However, miR376 a was rarely mentioned in these studies. We think this may be because its expression is limited to particular developmental stages or cell types.

In this study, we used human erythroleukemia K562 cells as an in vitro model of erythropoiesis because they have been proven to be a powerful tool for investigating erythriod cell development $[31,32]$. We found that miR376a is detectable in K562, but not in other leukemia cell lines. This kind of specificity of expression is observed for other regulators that are specifically expressed in certain phases or cell types and are involved in cell fate determination or lineage commitment. Furthermore, miR-376a expression was dramatically decreased in K562 cells and CD34+ HPCs during early erythroid dif- 
ferentiation, indicating that it may function as a negative regulator in this 'priming' process. Previous studies have reported a 'priming' state in the commitment process of HPCs to the erythroid lineage [13]. This priming state consists of various key events, such as the reprogramming of internal gene expression patterns and entrance into cell cycle. Notably, miR-376a expression changed markedly during the early stages of erythroid differentiation, suggesting that the specificity of miR-376a downregulation may contribute to the priming state of differentiation. The decline of miR-376a expression may unblock other functional proteins and promote erythropoiesis.

Ago2 is the only member of Argonaute family that has retained catalytic competence and it is uniquely required for viability in mice [33]. A well-known characteristic of Ago2 is its catalytic activity in the miRNA-directed cleavage of target mRNAs and in miRNA biogenesis [26, 34-36]. Homozygous Ago2 mutant mice demonstrate obvious anaemia and severe defects in erythropoiesis, and die shortly after birth [27]. The arrest in the maturation of erythroid precursors caused by Ago 2 deficiency can be explained by either its catalysis-independent or cleavagedependent roles in the transition of cells from progenitors to mature erythroblasts. However, the function of Ago2 in human erythropoiesis has not been reported previously. The results reported here suggest that Ago2 also participates in controlling human erythroid differentiation. Moreover, over-expression of miR-376a, via its ability to negatively regulate Ago 2 expression, may suppress erythroid differentiation through inhibition of Ago2, which is required for miRNA biogenesis. This is consistent with the report that Ago2 catalytic deficiency results in a strong reduction in miR-451, whose expression is indispensable to erythrocyte maturation [26, 27].

Cell proliferation and cell differentiation are delicately connected [37, 38]. They can either be coupled, as they are at the initiation of differentiation, or conflicting, as during terminal differentiation, when the cell withdraws from the cell cycle; this relationship is dependant on the precise stage of differentiation at which the cell division occurs $[24,39,40]$. An expansion phase during the early stages of erythroid differentiation, during which the quiescent progenitors are released to a proliferative state, is necessary for subsequent events in erythropoiesis [3, 41], and the high proliferation potential of early erythroblasts is associated with the temporal up-regulation of CDK2 $[24,42]$. In this study, we show that CDK2 is a direct target of miR-376a. Down-regulation of miR-376a after hemin treatment unblocks CDK2 expression and accelerates cell division in K562 cells, thus insuring the rapid proliferation of early erythroblasts. We reasoned that this highly proliferative state is needed for the committed progenitor to execute its subsequent differentiation program. In contrast, over-expression of miR-376a suppressed CDK2-dependent progenitor cell proliferation and resulted in arrested erythroid maturation. This is consistent with a previous report that the level of osteoblastic differentiation was lower when cell proliferation was inhibited [43]. Therefore, miR-376a modulates erythroid differentiation partly via interfering with cell cycle progression. Taken together, our results indicate that the erythroid-specific miR-376a regulates erythropoiesis in both K562 and HPCs by targeting the two ubiquitously expressed genes, $\mathrm{Ago} 2$ and $C D K 2$.

MiR-376a demonstrates relatively universal expression among different tissues, such as brain, kidney, bone marrow, heart, ovary, and stomach. However, only limited attention has been paid to this miRNA. This may be because it is difficult to detect and is only expressed in certain cell types or developmental stages. MiR-376a was initially cloned from pancreas cells and was shown to have increased expression in pancreatic cancer cells [44]. K562 is also a tumor cell line, which was established from a patient with chronic myologenous leukemia (CML) [31]. It was characterized as arising from a highly undifferentiated progenitor of the erythrocytic and megakaryocytic lineages [32]. The relatively high expression of miR-376a in K562 may partly contribute to the erythroid differentiation arrest and malignant transformation of these cells.

\section{Materials and Methods}

\section{Cell lines and cell culture}

The following human leukemia cell lines were used in this study: K562 (human chronic myelogenous leukemia cell line), HEL (human erythroleukemia cell line), HL-60 (human promyelocytic leukemia cell line), Jurkat (human acute T cell leukemia cell line), HUT-78 (human T lymphoma cell line), CMK (human megakaryoblastic leukemia cell line), 3D5 (human B cell line), Raji (human B-non-Hodgkin's lymphoma cell line), THP-1 (human monocytic leukemia cell line), and Hela (human epithelial carcinoma cell line). All of these cells were maintained in DMEM supplemented with $10 \%$ fetal bovine serum (GIBCO, NY, USA). For induction of erythroid differentiation in K562 cells, $30 \mu \mathrm{M}$ hemin (Sigma-Aldrich, Deisenhofen, Germany) was added to the medium for the duration of the experiment. Benzidine staining was used to detect hemoglobin-positive cells.

Oligonucleotides, constructs, cell transfection, and dual luciferase assays

MiRNA-376a precursor molecules (pre-miR-376a) and negative control molecules (scrambled dsRNAs) (AM17100) were obtained from Ambion (Ambion, TX, USA) and transfected, using Lipofectamine 2000 (Invitrogen, Carlsbad, CA, USA), into K562 cells at a final concentration of $60 \mathrm{nM}$. 
MiR-376a inhibitor molecules (Anti-miR-376a) and negative control molecules (negative control) (AM17010) were obtained from Ambion and used as above.

Ago2 siRNA, CDK2 siRNA, and negative control siRNA pools (D-001206-13-05) were synthesized by Dharmacon and transfected into K562 cells (200 nM) using Lipofectamine 2000. Cells were cultured for $48 \mathrm{~h}$ and harvested for western blot analysis as described below.

The reverse complementary sequence of miR-376a was inserted into pRL-TK downstream of the Renilla luciferase gene (Promega, WI, USA) to generate a reporter system (pRL-TK-miR-376a) to detect mature miR-376a expression in K562 and Hela cells. The 3' UTRs of human Ago2 and CDK2 mRNAs were PCR amplified and cloned into pRL-TK to generate the Ago2 reporter (PRL-TKAgo2) and CDK2 reporter (pRL-TK-CDK2). Mutations in these two mRNA sequences were created using the QuickChange SiteDirected Mutagenesis kit (Stratagene, CA, USA). The Hela and K562 cells were co-transfected with $0.4 \mu \mathrm{g}$ of the reporter construct, $0.2 \mu \mathrm{g}$ of pGL-3 control vector, and pre-miR-376a or negative controls. Cells were harvested $24 \mathrm{~h}$ post-transfection and assayed with Dual Luciferase Assay (Promega, WI, USA) according to manufacturer's instructions. All transfection assays were carried out in triplicate (Supplementary information, Table S1).

\section{Rescue assays of $A G O 2$ and $C D K 2$ gene expression}

The full length $A g o 2$ and $C D K 2$ cDNAs (which included the ORF and 3' UTR) were PCR amplified and cloned into pcDNA3.1 to generate the pcDNA-Ago 2 and pcDNA-CDK2 constructs, which were used in the rescue assays. K562 cells in 6-well plates were first transfected with pre-miR-376a or scrambled dsRNAs (60 $\mathrm{nM})$. After $24 \mathrm{~h}$ in culture, these K562 cells were then co-transfected with either pre-miR-376a $(30 \mathrm{nM})$ and $2.0 \mu \mathrm{g}$ pcDNA-Ago2/ pcDNA-CDK2, pre-miR-376a (30 nM) and $2.0 \mu \mathrm{g}$ pcDNA-empty, scrambled dsRNAs $(30 \mathrm{nM})$ and $2.0 \mu \mathrm{g}$ pcDNA-Ago2/ pcDNACDK2, or scrambled dsRNAs $(30 \mathrm{nM})$ and $2.0 \mu \mathrm{g}$ pcDNA-empty $12 \mathrm{~h}$ before hemin induction. Cells were harvested at indicated time points after hemin addition and assayed as required.

\section{$R N A$ isolation and quantitative real time $R T-P C R$}

Total RNA was extracted from the harvested cells using Trizol reagent (Invitrogen, CA, USA) according to the manufacturer's instructions. Small RNAs ( <200 nt) were extracted from cells using the mirVana miRNA Extraction Kit (Ambion, TX, USA) according to the manufacturer's instructions.

The RNA was quantified by absorbance at $260 \mathrm{~nm}$. cDNA was synthesized from $2 \mu \mathrm{g}$ of total RNA or $20 \mathrm{ng}$ of small RNA using M-MLV reverse transcriptase (Invitrogen). Oligo (dT) ${ }_{18}$ were used as the RT primers for the reverse transcription of mRNAs. According to Chen's report, stem-loop RT primers were used for the reverse transcription of miRNAs [45]. Quantitative RT-PCR was carried out using the ABI PRISM 7500 real-time PCR System (Applied Biosystems, CA, USA) using the SYBR Premix Ex Taq kit (Takara, Dalian, China) according to the manufacturer's instructions. For mRNAs, the data were normalized using endogenous $G A P D H$ as a control. For miRNAs, U6 snRNA was used as the endogenous control. The comparative $\mathrm{Ct}$ method was used to quantify target genes relative to their endogenous control. For each individual analysis, one of the samples was designated as the calibrator and given a relative value of 1.0. All quantities were ex- pressed as n-fold relative to the calibrator.

\section{Northern blots and western blots}

Northern blot analysis of miRNAs was performed as described previously [21]. Western blot analysis was performed as described previously [46] and membranes were blotted with antibodies for Ago2 (Millipore, MA, USA), CDK2 (Becton-Dickinson, NJ, USA), RB, phosphorylated RB (p-RB), and GAPDH (Abcam, Cambridge, UK). Immunoblots were quantified by ImageJ software.

\section{Flow cytometry}

For the detection of intracellular DNA, harvested cells were washed twice with PBS and fixed in $75 \%$ ethanol at $4{ }^{\circ} \mathrm{C}$ overnight. Following two washes with ice-cold PBS, the fixed cells were incubated in RNaseA $(20 \mu \mathrm{g} / \mathrm{mL})$ at $37^{\circ} \mathrm{C}$ for $30 \mathrm{~m}$, and then stained with PI (propidium iodide) $(0.5 \mathrm{mg} / \mathrm{mL})$ at $4{ }^{\circ} \mathrm{C}$ for 30 min. Cells were washed with BSA/PBS $(1 \%)$ and resuspended in $500 \mu \mathrm{l}$ PBS. Flow cytometric data detecting PI were acquired from about $10^{5}$ cells using the Becton-Dickinson flow cytometer. All assays were carried out in triplicate. The average percentages of cells in $\mathrm{G} 1$ and $\mathrm{S}$ phase are shown in Supplementary information, Table $\mathrm{S} 2$ (corresponding to Figure 5F) and Supplementary information, Table S3 (corresponding to Figure 6B).

Isolation and erythroid induction culture of CD34+ hematopoietic stem/progenitor cells

Human umbilical cord blood (UCB) was obtained from normal full-term deliveries after informed consent as approved by the Research Ethics Committee of the Millitary General Hospital of Beijing (Beijing, China). Mononuclear cell (MNC) fractions were isolated from UCB by percoll density gradient $(\mathrm{d}=1.077$; Amersham Biotech, Germany). CD34+ cells were enriched from MNCs through positive immunomagnetic selection (CD34 MultiSort kit, Miltenyi Biotec, Bergisch-Glad-bach, Germany). The purity of the CD34+ cells isolated by this isolation system was more than $90 \%$. The CD34+ cells were cultured in IMDM supplemented with $30 \%$ fetal bovine serum (GIBCO, NY, USA), 1\% BSA, $100 \mu \mathrm{M}$ 2-ME, $2 \mathrm{ng} / \mathrm{mL}$ recombinant human IL-3, $100 \mathrm{ng} / \mathrm{mL}$ recombinant human SCF (PeproTech, London, UK), 2U/mL recombinant human EPO (R\&D Systems, MN, USA), $60 \mathrm{mg} / \mathrm{mL}$ penicillin, and $100 \mathrm{mg} / \mathrm{mL}$ streptomycin. Cells were harvested every 3-5 days.

\section{Lentivirus infection}

The self-inactivating transfer vector plasmid containing miR376a (pMIR-376a) and the packaging kit were purchased from System Biosciences (SBI, CA, USA) and used according to the manufacturer's instructions. The recombinant lentivirus particles (lenti-miR-376a and control lenti-vector) were harvested and added to the CD34+ cell culture medium. Cells were washed the next day with PBS and plated for colony-forming experiments and liquid cultures.

\section{Proliferation assay}

To measure the effect of miR-376a on cellular proliferation rates, K562 cells were transfected with pre-miR-376a or control precursor and cultured in normal culture media at $37^{\circ} \mathrm{C}$. CD34+ HPCs were infected using lenti-miR-376a or lenti-vector and cultured in liquid medium. The percentage of viable cells was deter- 
mined by trypan blue staining at 24,48 , and $72 \mathrm{~h}$ post-transfection, or $3,5,10$, and 14 days post-infection.

\section{Colony forming assay and Giemsa staining}

The colony forming cell assay was performed in triplicate using human methylcellulose media (R\&D Systems, MN, USA) according to the manufacturer's instructions. Human CD34+ cells were cultured in $35-\mathrm{mm}$ plates with medium containing $1.3 \%$ methylcellulose, 25\% FBS, $2 \%$ bovine serum albumin, $2 \mathrm{mmol} / \mathrm{L}$ L-glutamine, $0.05 \mathrm{mmol} / \mathrm{L} 2$-mercaptoethanol, $50 \mathrm{ng} / \mathrm{mL}$ stem cell factor (SCF), $10 \mathrm{ng} / \mathrm{mL}$ interleukin-3 (IL-3), and $2 \mathrm{U} / \mathrm{mL}$ erythropoietin (EPO). To quantify the number of CFU-Es colonies, the dishes were examined for hemoglobinized and colonies with 8 or more cells on day 7 of culture. BFU-Es was scored on day 14 and identified as large aggregates of 64 or more hemoglobinized cells, or as clusters of 3 or more subcolonies with 8 or more hemoglobinized cells per subcolony. For morphological analysis, cells were smeared on glass slides by cytopin centrifugation, stained with Giemsa, and analyzed at $400 \times$ magnification under a microscope (Nikon TE2000) equipped with a digital camera.

\section{Statistics}

Student's $t$-test (two-tailed) was performed to analyze the data. $P$-values $<0.05$ were considered significant.

\section{Acknowledgments}

The authors thank Drs Hua Bai and Cuimin Lu from the Millitary General Hospital of Beijing for assistance in umbilical cord blood collection. This work was supported by grants from the National Natural Science Foundation of China (30871249 and 30721063); the Specific Fund of National Laboratory of China (3060204); and IBMS, CAMS (2009RC03).

\section{References}

1 Gregory CJ, Eaves AC. Three stages of erythropoietic progenitor cell differentiation distinguished by a number of physical and biologic properties. Blood 1978; 51:527-537.

2 Stamatoyannopoulos G. Control of globin gene expression during development and erythroid differentiation. Exp Hematol 2005; 33:259-271.

3 Migliaccio AR, Migliaccio G. The control of proliferation and differentiation of early erythroid progenitors. Biotherapy 1990; 2:299-303.

4 Ambros V. microRNAs: tiny regulators with great potential. Cell 2001; 107:823-826.

5 Di Leva G, Calin GA, Croce CM. MicroRNAs: fundamental facts and involvement in human diseases. Birth Defects Res C Embryo Today 2006; 78:180-189.

6 Guarnieri DJ, DiLeone RJ. MicroRNAs: a new class of gene regulators. Ann Med 2008; 40:197-208.

7 Shivdasani RA. MicroRNAs: regulators of gene expression and cell differentiation. Blood 2006; 108:3646-3653.

8 Kluiver J, Kroesen BJ, Poppema S, van den Berg A. The role of microRNAs in normal hematopoiesis and hematopoietic malignancies. Leukemia 2006; 20:1931-1936.

9 Vasilatou D, Papageorgiou S, Pappa V, Papageorgiou E, Der- venoulas $\mathrm{J}$. The role of microRNAs in normal and malignant hematopoiesis. Eur J Haematol 2010; 84:1-16.

10 Fazi F, Rosa A, Fatica A, et al. A minicircuitry comprised of microRNA-223 and transcription factors NFI-A and C/EBPalpha regulates human granulopoiesis. Cell 2005; 123:819-831.

11 Georgantas RW 3rd, Hildreth R, Morisot S, et al. CD34+ hematopoietic stem-progenitor cell microRNA expression and function: a circuit diagram of differentiation control. Proc Natl Acad Sci USA 2007; 104:2750-2755.

12 Felli N, Fontana L, Pelosi E, et al. MicroRNAs 221 and 222 inhibit normal erythropoiesis and erythroleukemic cell growth via kit receptor down-modulation. Proc Natl Acad Sci USA 2005; 102:18081-18086.

13 Masaki S, Ohtsuka R, Abe Y, Muta K, Umemura T. Expression patterns of microRNAs 155 and 451 during normal human erythropoiesis. Biochem Biophys Res Commun 2007; 364:509-514.

14 Pase L, Layton JE, Kloosterman WP, Carradice D, Waterhouse PM, Lieschke GJ. miR-451 regulates zebrafish erythroid maturation in vivo via its target gata2. Blood 2009; 113:1794-1804.

15 Bruchova-Votavova H, Yoon D, Prchal JT. miR-451 enhances erythroid differentiation in K562 cells. Leuk Lymphoma 2010; 51:686-693.

16 Bianchi N, Zuccato C, Lampronti I, Borgatti M, Gambari R. Expression of miR-210 during erythroid differentiation and induction of gamma-globin gene expression. BMB Rep 2009; 42:493-499.

17 Noh SJ, Miller SH, Lee YT, et al. Let-7 microRNAs are developmentally regulated in circulating human erythroid cells. J Transl Med 2009; 7:98.

18 Yuan JY, Wang F, Yu J, Yang GH, Liu XL, Zhang JW. MicroRNA-223 reversibly regulates erythroid and megakaryocytic differentiation of K562 cells. J Cell Mol Med 2009; 13:4551-4559.

19 Felli N, Pedini F, Romania P, et al. MicroRNA 223-dependent expression of LMO2 regulates normal erythropoiesis. Haematologica 2009; 94:479-486.

20 Yang GH, Wang F, Yu J, Wang XS, Yuan JY, Zhang JW. MicroRNAs are involved in erythroid differentiation control. $J$ Cell Biochem 2009; 107:548-556.

21 Yu J, Wang F, Yang GH, et al. Human microRNA clusters: genomic organization and expression profile in leukemia cell lines. Biochem Biophys Res Commun 2006; 349:59-68.

22 O'Carroll D, Mecklenbrauker I, Das PP, et al. A Slicerindependent role for Argonaute 2 in hematopoiesis and the microRNA pathway. Genes Dev 2007; 21:1983-1988.

23 Martinez J, Busslinger M. Life beyond cleavage: the case of Ago2 and hematopoiesis. Genes Dev 2007; 21:1999-2004.

24 Dai MS, Mantel CR, Xia ZB, Broxmeyer HE, Lu L. An expansion phase precedes terminal erythroid differentiation of hematopoietic progenitor cells from cord blood in vitro and is associated with up-regulation of cyclin E and cyclin-dependent kinase 2. Blood 2000; 96:3985-3987.

25 Donker RB, Mouillet JF, Nelson DM, Sadovsky Y. The expression of Argonaute2 and related microRNA biogenesis proteins in normal and hypoxic trophoblasts. Mol Hum Reprod 2007; 13:273-279.

26 Diederichs S, Haber DA. Dual role for argonautes in mi- 
croRNA processing and posttranscriptional regulation of microRNA expression. Cell 2007; 131:1097-1108.

27 Cheloufi S, Dos Santos CO, Chong MM, Hannon GJ. A dicerindependent miRNA biogenesis pathway that requires Ago catalysis. Nature 2010; 486:584-589.

28 Cifuentes D, Xue H, Taylor DW, et al. A Novel miRNA Processing Pathway Independent of Dicer Requires Argonaute2 Catalytic Activity. Science 2010; 328:1694-1698.

29 Liu C, Yu J, Yu S, et al. MicroRNA-21 acts as an oncomir through multiple targets in human hepatocellular carcinoma. $J$ Hepatol 2010; 53:98-107.

30 Kucukkaya B, Arslan DO, Kan B. Role of G proteins and ERK activation in hemin-induced erythroid differentiation of K562 cells. Life Sci 2006; 78:1217-1224.

31 Gahmberg CG, Andersson LC. K562--a human leukemia cell line with erythroid features. Semin Hematol 1981; 18:72-77.

32 Rowley PT, Ohlsson-Wilhelm BM, Farley BA, LaBella S. Inducers of erythroid differentiation in K562 human leukemia cells. Exp Hematol 1981; 9:32-37.

33 Alisch RS, Jin P, Epstein M, Caspary T, Warren ST. Argonaute2 is essential for mammalian gastrulation and proper mesoderm formation. PLoS Genet 2007; 3:e227.

34 Förstemann K, Horwich MD, Wee L, Tomari Y, Zamore PD. Drosophila microRNAs are sorted into functionally distinct argonaute complexes after production by dicer-1. Cell 2007; 130:287-297.

35 Bhattacharyya SN, Filipowicz W. Argonautes and company: sailing against the wind. Cell 2007; 128:1027-1028.

36 Kawamura Y, Saito K, Kin T, et al. Drosophila endogenous small RNAs bind to Argonaute 2 in somatic cells. Nature
2008; 453:793-797.

37 Maione R, Amati P. Interdependence between muscle differentiation and cell-cycle control. Biochim Biophys Acta 1997; 1332:M19-M30.

38 Walsh K, Perlman H. Cell cycle exit upon myogenic differentiation. Curr Opin Genet Dev 1997; 7:597-602.

39 Hsieh FF, Barnett LA, Green WF, et al. Cell cycle exit during terminal erythroid differentiation is associated with accumulation of p27(Kip1) and inactivation of cdk2 kinase. Blood 2000; 96:2746-2754.

40 Kiyokawa H, Kineman RD, Manova-Todorova KO, et al. Enhanced growth of mice lacking the cyclin-dependent kinase inhibitor function of p27(Kip1). Cell 1996; 85:721-732.

41 Malumbres M, Barbacid M. To cycle or not to cycle: a critical decision in cancer. Nat Rev Cancer 2001; 1:222-231.

42 Furukawa Y. Cell cycle control genes and hematopoietic cell differentiation. Leuk Lymphoma 2002; 43:225-231

43 Mizuno Y, Yagi K, Tokuzawa Y, et al. miR-125b inhibits osteoblastic differentiation by down-regulation of cell proliferation. Biochem Biophys Res Commun 2008; 368:267-272.

44 Poy MN, Eliasson L, Krutzfeldt J, et al. A pancreatic isletspecific microRNA regulates insulin secretion. Nature 2004; 432:226-230.

45 Chen C, Ridzon DA, Broomer AJ, et al. Real-time quantification of microRNAs by stem-loop RT-PCR. Nucleic Acids Res 2005; 33:e179.

46 Yu J, Ryan DG, Getsios S, Oliveira-Fernandes M, Fatima A, Lavker RM. MicroRNA-184 antagonizes microRNA-205 to maintain SHIP2 levels in epithelia. Proc Natl Acad Sci USA 2008; 105:19300-19305.

(Supplementary information is linked to the online version of the paper on the Cell Research website.) 